$\begin{array}{cc}\text { ACADEMIA ROMÂNĂ } & \text { Rev. Roum. Chim., } \\ \text { 2021, 66(2), 193-198 } \\ \text { Revue Roumaine de Chimie } \\ \text { http://web.icf.ro/rrch/ }\end{array}$

\title{
INVESTIGATION OF SURFACE RELIEF GRATINGS ON AZO-COPOLYIMIDE FILMS USING ATOMIC FORCE MICROSCOPY
}

\author{
Ion SAVA, ${ }^{\mathrm{a}^{*}}$ Iuliana STOICA, ${ }^{\mathrm{a}}$ Ilarion MIHAILA ${ }^{\mathrm{b}}$ and Ionut TOPALA ${ }^{\mathrm{c}}$ \\ a "Petru Poni" Institute of Macromolecular Chemistry, Iaşi - 700487, Roumania \\ ${ }^{b}$ Integrated Center of Environmental Science Studies N-E Development Region (CERNESIM), \\ "Alexandru Ioan Cuza" University of Iaşi, Iaşi - 700506, Roumania \\ ${ }^{\mathrm{c}}$ Plasma Advanced Research Center (IPARC), Faculty of Physics, \\ "Alexandru Ioan Cuza" University of Iaşi, Iaşi - 700506, Roumania
}

Azo-copolyimide films were explored regarding the surface relief gratings formation under the action of pulsatory laser irradiation. The azo-copolyimides have been synthesized by the polycondensation reaction of benzophenonetetracarboxylic dianhydride and a mixture of two diamines, one of which contains substituted phenyl groups and the other one contains two pendent substituted azobenzene group, taken in the molar ratio $3: 1$, respectively. Atomic force microscopy was used to investigate the surface relief gratings characteristics in different conditions and for different structures of the azo-copolyimide films. (b)
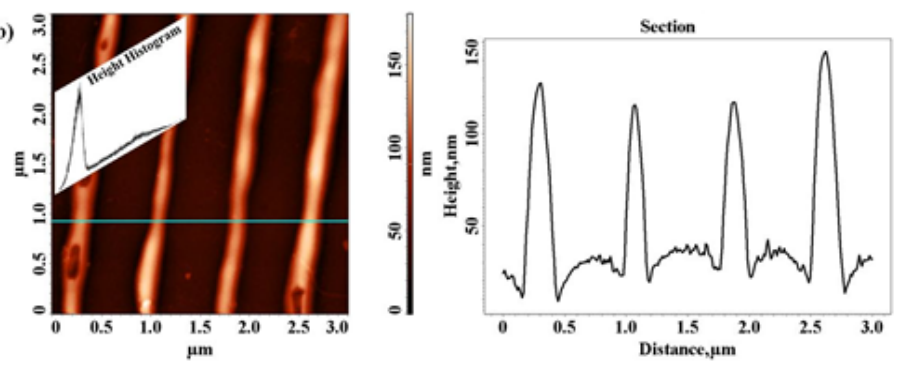

\section{INTRODUCTION}

Considerable interest have been made in the development of materials that are optically responsive and have the potential to be useful in photonic technology. Azobenzene and many of its derivatives are promising materials for such applications. The utility of these materials is based on multiple trans-cis-trans isomerization cycles of azobenzene groups accompanied by their movement and rearrangement of the macromolecular chains to the laser polarization beam. ${ }^{1,2}$ Polymers with such properties have shown potential technological applications such as optical information storage and processing, optical switching devices, holographic gratings, fabrication of diffractive elements with specific polarization properties, waveguides and many others. ${ }^{1-8}$ For advanced practical applications, the azopolymers should fulfill other requirements like short response time, long-term chemical and orientational stabilities at the operating temperatures, good film forming ability, high thermal stability and good processability. The physical and photoinduced properties are strongly associated, with both the chromophore and polymer matrix architecture. The more vulnerable part in such materials is the azobenzene group. To

\footnotetext{
${ }^{*}$ Corresponding author: isava@icmpp.ro
} 
increase the thermal stability of optically induced birefringence the combination of high glass transition temperature ( $\mathrm{Tg}$ ) polymers, such as polyimides, was used by many authors. ${ }^{9-14}$

The incorporation of azobenzene chromophore into the polymers can introduce structural diversity and processability into the functional materials. Laser-induced surface relief gratings on azobenzene polymer films have attracted considerable attention since first reported by different authors. ${ }^{3,4}$ The formed surface relief gratings are stable when kept below glass transition temperature. They will erased by laser or heating. ${ }^{15,16}$ The atomic force microscope (AFM) is a powerful device to characterize and imaging the nanomaterial surfaces. AFM as a unique tool not only can be used to study the topography and morphology of films, but also applied to probe the local mechanical behavior of films, especially the force response to approach. Many researchers have been focused on the progress in AFM techniques to characterize the films, single cells and single molecules in nano-scale. ${ }^{17,18}$

The investigation of surface relief gratings (SRG) was performed by using atomic force microscopy to analyse the nanochannels characteristics which were obtained on the film surfaces. This work studies by AFM the structuring behaviour of polymer films which contain in the same macromolecular chain a rigid part and a flexible part. The rigid part consist in a matrix of aromatic polyimide and a flexible part was introduced in a side azobenzene group containing different lengths of alkyl units.

\section{RESULTS AND DISCUSSION}

\section{Surface relief gratings of azo-copolyimide films}

The parameters used for obtaining surface relief gratings (SRG) on azo-copolyimides films were choose based on our previously study performed on other azo-polyimides and azo-copolyimides. ${ }^{11-}$ 14, 16,19,20 The investigations of surface structuring capacity have been performed by using a laser field with an energy density of $45 \mathrm{~mJ} / \mathrm{cm}^{2}$ and two different number of pulses, 10 and 100 . We try to use a lower energy density (i.e. $10 \mathrm{~mJ} / \mathrm{cm}^{2}$ ) but no SRG were obtained on surface of the polymer films. Surface irradiation was realised under the action of a pulsed laser field, with a controlled distribution of energy represented by an interference pattern after passing through the phase mask. The energy source is represented by $6 \mathrm{~ns}$ $\mathrm{Nd}$ :YAG laser pulses working on his third harmonic at $355 \mathrm{~nm}$.

Atomic force microscopy was used to characterize the resulted structured surfaces. The bimodal distribution of heights (height histograms) corresponding to the AFM morphological images from Figs. 1-4 indicates the existence over the surface of two different entities, i.e. the two regions of the SRGs. The lengths of the alkyl chains have established the pattern depth, calculated using the cross-section profiles corresponding to the AFM morphological images (Figs. 1-4). The obtained results presented in Table 1 show that by increasing the number of alkyl chain in the macromolecular chain of the azocopolyimides, the modulation depth decrease, irrespective of the pulses number used. Thus, by using 10 pulses number, for azo-copolyimide CPI 1,3 which incorporate in the azobenzene side linkage three methylene units, the modulation depth was $134 \pm 8 \mathrm{~nm}$. For azo-copolyimide CPI 1,4 which contains four methylene units the modulation depth was $96 \pm 6 \mathrm{~nm}$ and for CPI-1,5 having five methylene units and CPI 1,6 which incorporate six methylene units the modulation depth decrease to $91 \pm 5 \mathrm{~nm}$ and $88 \pm 7 \mathrm{~nm}$, respectively. It seems that the flexibility of the azo-copolyimide with a small number of alkyl chains is greater, inducing a greater structuring capacity, and thus a more pronounced relief. By increasing the pulses number to 100 , the generated channels were even and uniform as can be seen in Figs. 1-4, the modulation depth being significantly higher. By analyzing a profile diagram it was observed that the homogeneity of the surface features is satisfactory, the deviations being in the domain of 15-30 nm. Also, it was observed that the values of modulation depth decrease with increasing the number of methylene units in the side chains of the azobenzene groups, as can be seen in Table 1. The surface morphology has a preferential orientation, indicated by the texture direction index (Stdi), as can be seen in the AFM images presented in Figs. 1-4. A good anisotropy of the morphology of the SRG of the azocopolyimide films is evidenced by a low value of Stdi. ${ }^{21}$ As can be seen in Table 1 the values of Stdi are in the range of $0.183-0.367$. 
(a)

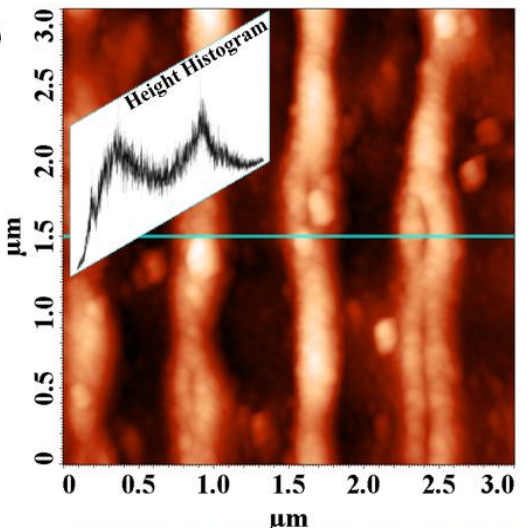

(b)

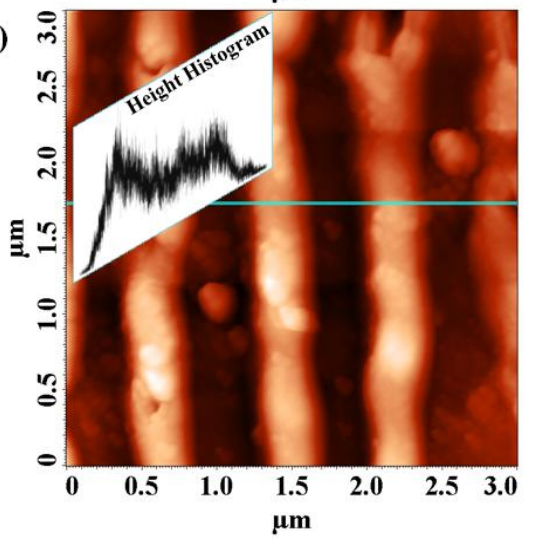

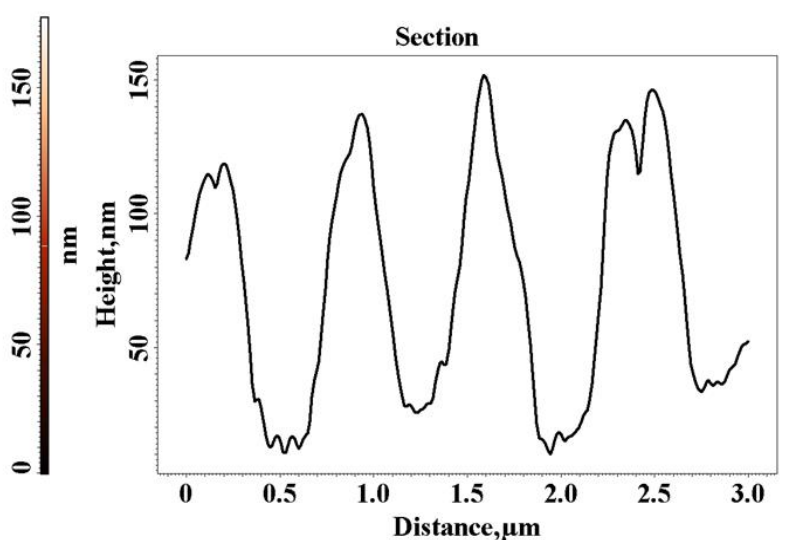

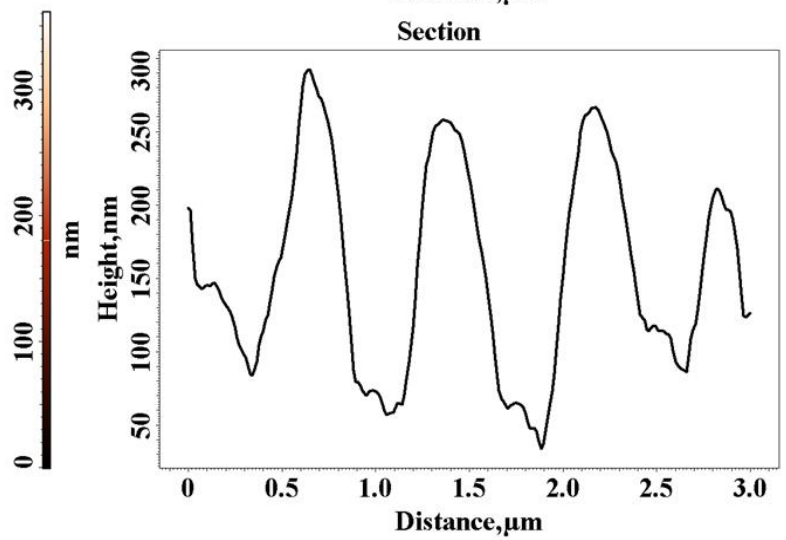

Fig. 1 - SRG of azo-copolyimide CPI 1,3 at 10 (a) and 100 (b) irradiation pulses.

(a)

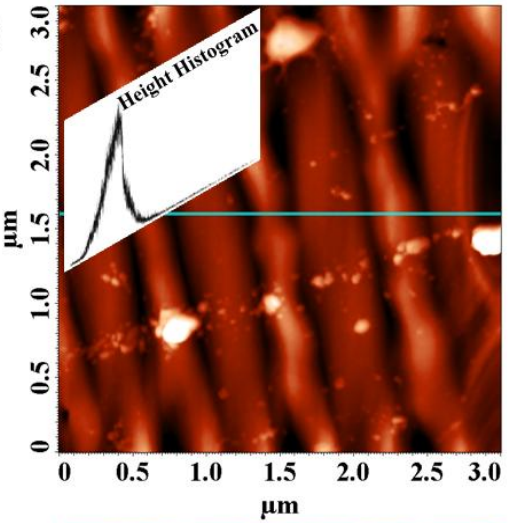

(b)

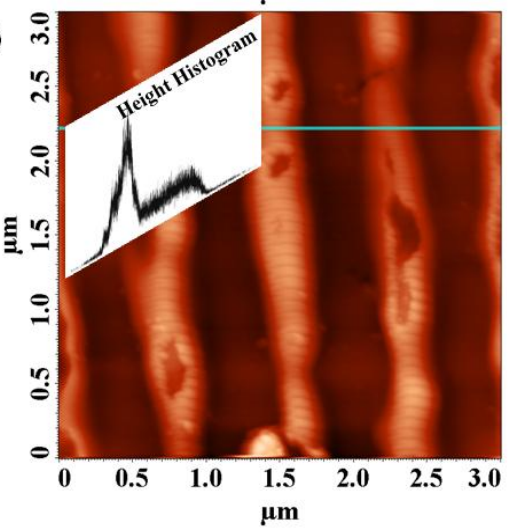

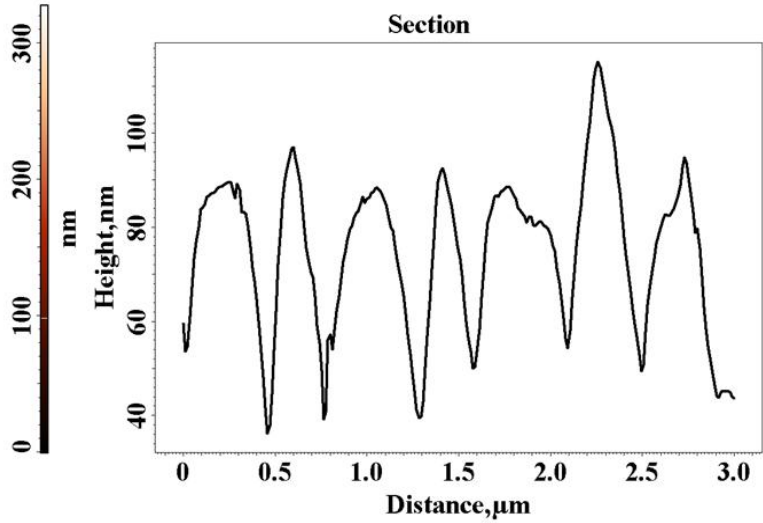

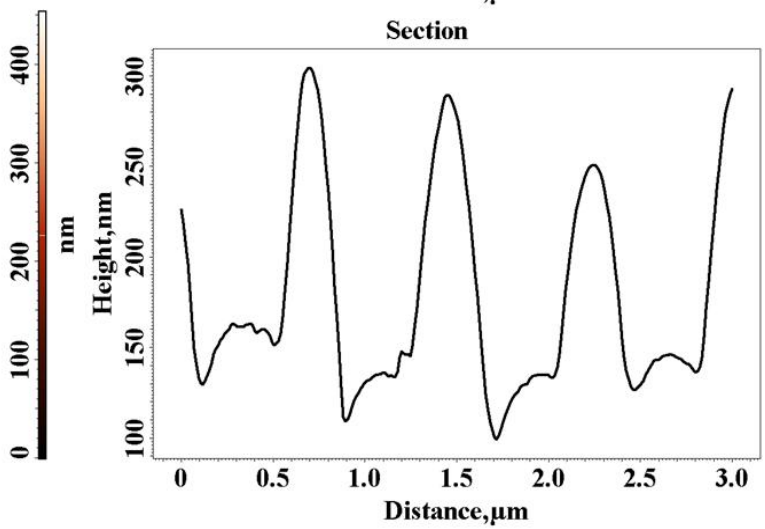

Fig. 2 - SRG of azo-copolyimide CPI 1,4 at 10 (a) and 100 (b) irradiation pulses. 
(a)

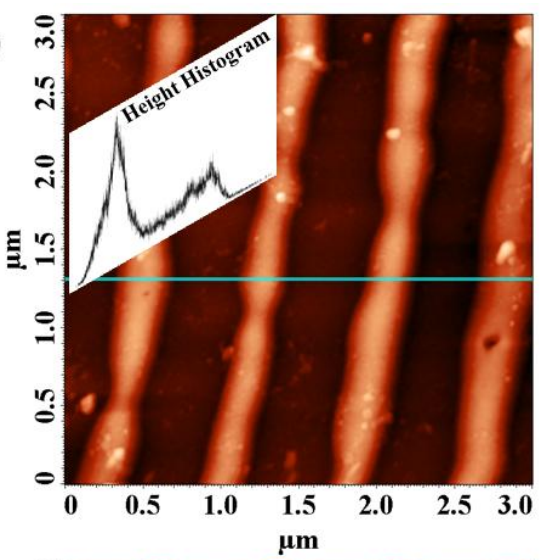

(b)

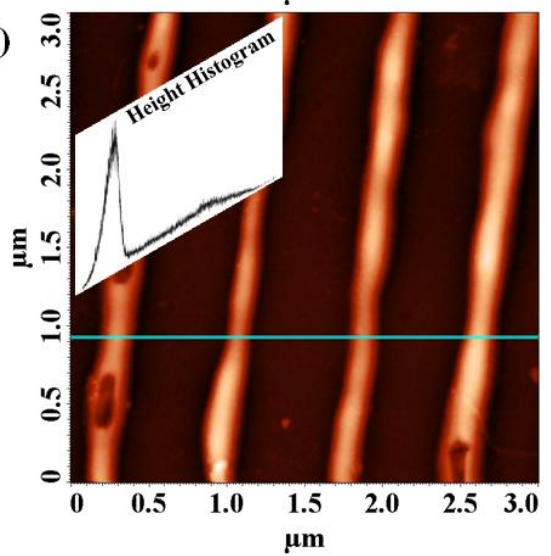

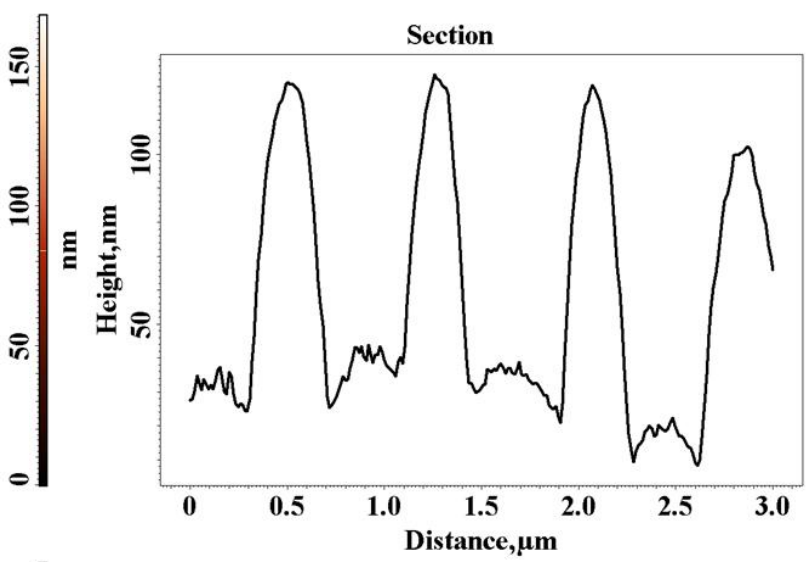

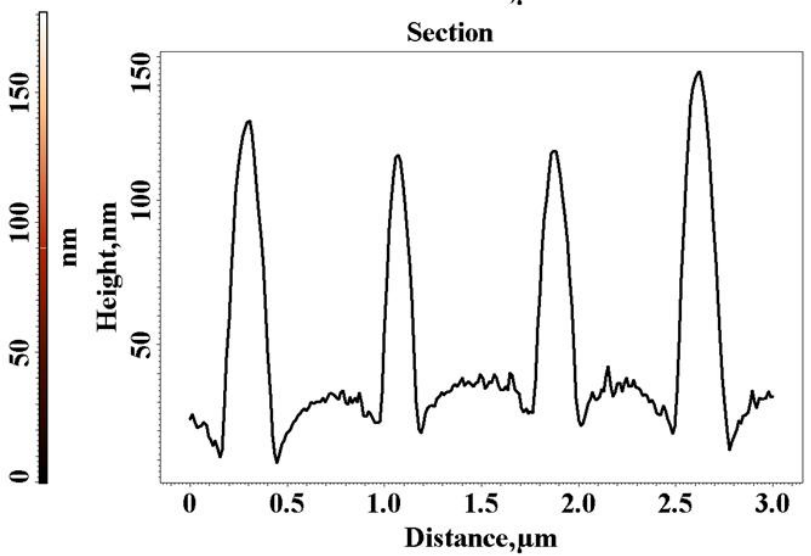

Fig. 3 - SRG of azo-copolyimide CPI 1,5 at 10 (a) and 100 (b) irradiation pulses.

(a)

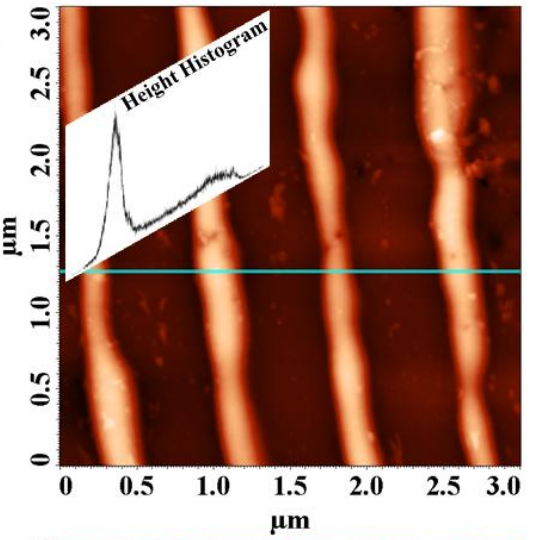

(b)

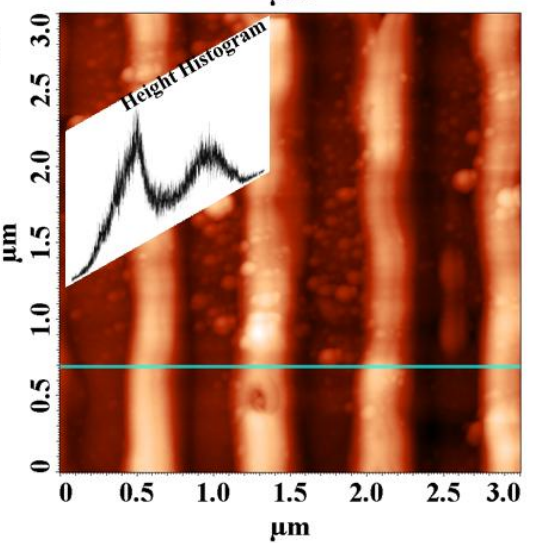

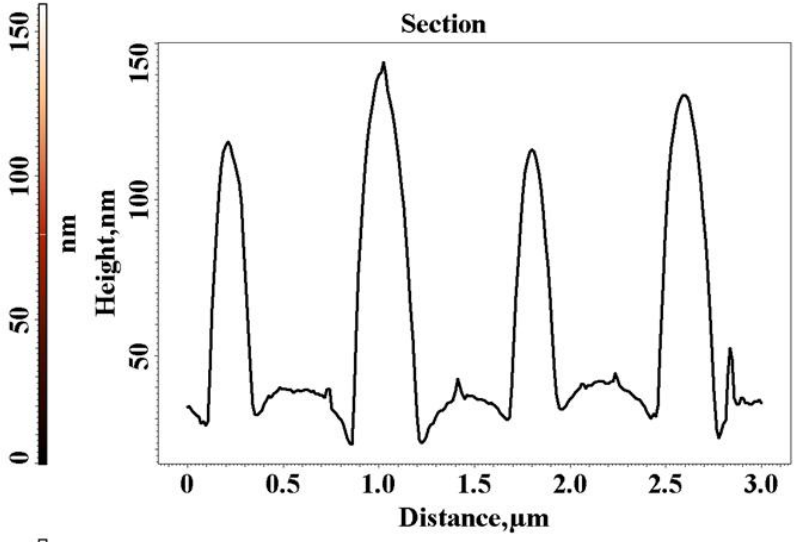

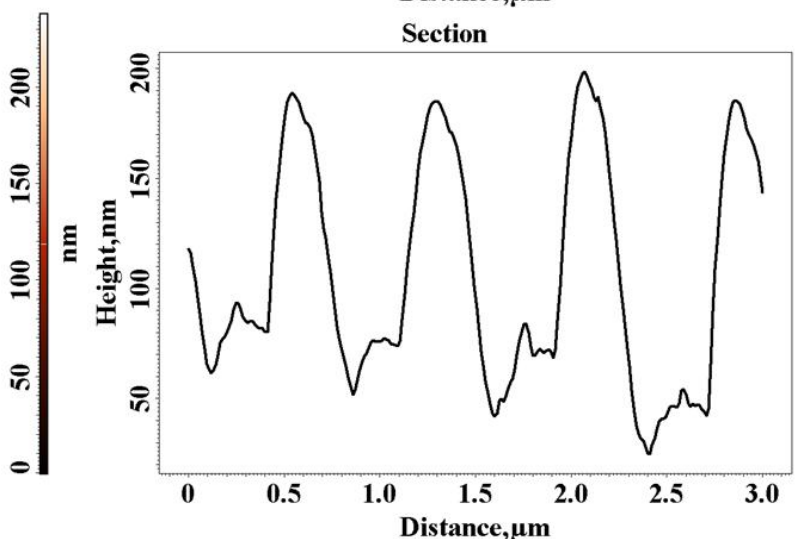

Fig. 4 - SRG of azo-copolyimide CPI 1,6 at 10 (a) and 100 (b) irradiation pulses. 
Table 1

The modulation depth and texture direction index of the azo-copolyimides

\begin{tabular}{|c|c|c|c|c|c|c|c|c|}
\hline \multirow{3}{*}{$\begin{array}{l}\text { Pulses } \\
\text { number }\end{array}$} & \multicolumn{8}{|c|}{ Azo-copolyimides } \\
\hline & \multicolumn{2}{|c|}{ CPI 1,3 } & \multicolumn{2}{|c|}{ CPI 1,4 } & \multicolumn{2}{|c|}{ CPI 1,5} & \multicolumn{2}{|c|}{ CPI 1,6 } \\
\hline & $\mathbf{Z}^{*}, \mathrm{~nm}$ & Stdi & $\mathbf{Z}^{*}, \mathrm{~nm}$ & Stdi & $\mathbf{Z}^{*}, \mathrm{~nm}$ & Stdi & $\mathbf{Z}^{*}, \mathrm{~nm}$ & Stdi \\
\hline 10 & $134 \pm 8$ & 0.274 & $96 \pm 6$ & 0.367 & $91 \pm 5$ & 0.299 & $88 \pm 7$ & 0.244 \\
\hline 100 & $221 \pm 31$ & 0.266 & $162 \pm 22$ & 0.310 & $145 \pm 14$ & 0.210 & $143 \pm 16$ & 0.183 \\
\hline
\end{tabular}

$\mathbf{Z}^{*}$ - Modulation depth; Stdi - texture direction index

The presence of two side azobenzene groups determined the structuring ability of these azocopolyimides. The presence of the BTDA units in the macromolecular chain of the azo-copolyimides slightly increase the rigidity of the chains but the presence of two side azobenzene units in the chain connected by ether and alkyl units improve the flexibility. The presence of MMDA have a small influence of the structuring capacity of the polymer films compared to aromatic diamines which contain ether linkages connected in meta or para position. ${ }^{11,16}$

\section{EXPERIMENTAL}

The structure of azo-copolyimides used to investigate the behavior to the laser patterning is shown in the Scheme 1 . These azo-copolymers have been synthesized by the polycondensation reaction of benzophenonetetracarboxylic dianhydride (BTDA) and a mixture of two diamines, one of which contains substituted phenyl groups, namely 4,4'diamino-3,3'-dimethyldiphenylmethane, (MMDA) and the other one contains two pendent substituted azobenzene group, namely 2,2 bis-(3-amino-4-alkyloxy-phenyl-4'-methylazobenzene) hexafluoropropane. The molar ratio between the two diamines was $3: 1$, respectively. The polycondensation reaction of these monomers and the details of the synthesis and characterization was carried out by using a procedure previously reported. ${ }^{22,23}$

The third harmonic (355 $\mathrm{nm}$ wavelength) of a pulsed Nd:YAG laser (Brilliant B from Quantel, 6 ns pulse duration, $5 \mathrm{~mm}$ diameter) was selected to generate an interference field in the proximity of a phase mask (1000 grooves per $\mathrm{mm}$ ), placed prior to the azo-copolyimides samples. The incident fluency on the grating was kept constant to $45 \mathrm{~mJ} / \mathrm{cm}^{2}$, while the laser pulses number was set to 10 and 100 .

The surface relief gratings were investigated by Atomic Force Microscopy by using a Scanning Probe Microscope Solver PRO-M (NT-MDT, Zelenograd, Russia). The film topography was analyzed in tapping mode, in air, using a rectangular cantilever NSG10/Au (NT-MDT, Zelenograd, Russia) with a resonant frequency of $277 \mathrm{kHz}$. Different scan sizes were analyzed by means of Nova v.1.26.0.1443 for Solver software, but the morphological features were easily observed when the scan length of $3 \mu \mathrm{m}$ was utilized. The tridimensional parameters were calculated using Image Analysis 3.5.0.18542 software.

\section{CONCLUSIONS}

Atomic force microscopy was used to investigate surface relief gratings formation under the action of pulsatory laser irradiation of the azocopolyimide films. All the azo-copolyimide films showed a good surface structuration capacity irrespective of pulsess number at $45 \mathrm{~mJ} / \mathrm{cm}^{2}$. The azo-copolyimides with a small number of alkyl chains have presented a more pronounced relief due to their flexibility. By using a high number of pulses, the surface relief grating became much more regular and the modulation depths in these cases were around 140-250 nm, while in the cases using only 10 irradiation pulses, the modulation depths were in the range of $80-150 \mathrm{~nm}$. The surface morphology has a preferential orientation, indicated by the low value of the texture direction index.

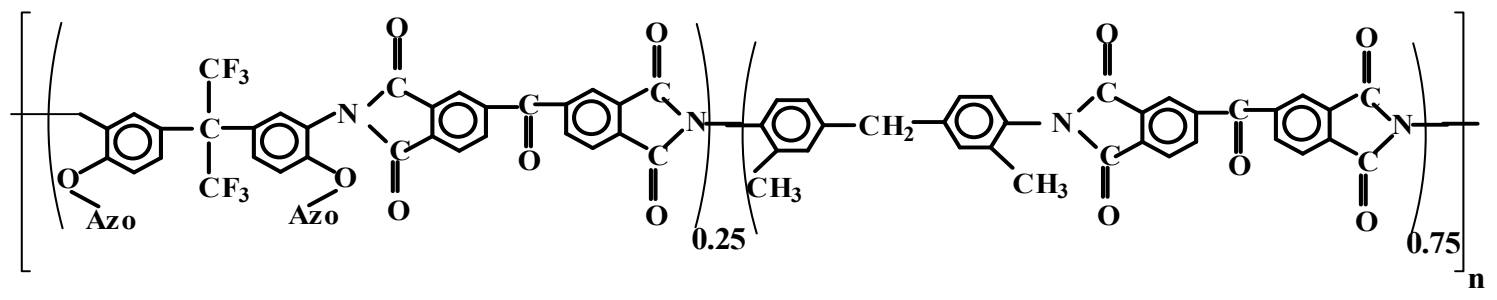

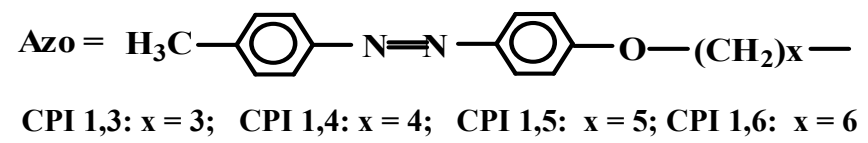

Scheme 1 - Structure of azo-copolyimides. 


\section{REFERENCES}

1. G. S. Kumar and D. C. Neckers, Chem. Rev., 1989, 89, 1915-1925.

2. L. Ding and T. P. Russell, Macromolecules, 2007, 40, 2267-2270.

3. P. Rochon, E. Batalla and A. Natansohn, Appl. Phys. Lett., 1995, 66, 136-138.

4. D. Y. Kim, S. K. Tripathy, L. Li and J. Kumar Appl. Phys. Lett., 1995, 66, 1166-1168.

5. J. A. Delaire and K. Nakatani, Chem. Rev., 2000, 100, 1817-1846.

6. A. Natansohn and P. Rochon, Chem. Rev., 2002, 102, 4139-4176.

7. D. Wang and X. Wang, Prog. Polym. Sci., 2013, 38, 271-301.

8. J. Konieczkowska, A. Kozanecka-Szmigiel, W. Piecek, R. Weglowski and E. Schab-Balcerzak, Polimery, 2018, 63, 481-487.

9. E. Schab-Balcerzak, B. Sapich and J. Stumpe, Polymer, 2005, 46, 49-59.

10. I. Sava, A. M. Resmerita, G. Lisa, V. Damian and N. Hurduc, Polymer, 2008, 49, 1475-1482.

11. I. Sava, A. Burescu, I. Stoica, V. Musteata, M. Cristea, I. Mihaila, V. Pohoata and I. Topala, $R S C A d v$., 2015, 5 , 10125-10133.
12. I. Sava, I. Stoica, I. Mihaila, V. Pohoata, I. Topala, G. Stoian and N. Lupu, Polym. Test. 2018, 72, 407-415.

13. J. Konieczkowska, E. Schab-Balcerzak, M. Libera, I. Mihaila and I. Sava, Eur. Polym. J., 2019, 110, 85-89.

14. K. Bujak, I. Sava, I. Stoica, V. Tiron, I. Topala, R. Węgłowski, E. Schab-Balcerzak and J. Konieczkowska, Eur. Polym. J., 2020, 126, 109563.

15. M. S. Ho, C. Barret, J. Paterson, M. Esteghamatian, A. Natansohn and P. Rochon, Macromolecules, 1996, 29, 4613-4619.

16. I. Sava, L. Sacarescu, I. Stoica, I. Apostol, V. Damian and N. Hurduc, Polym. Int., 2009, 58, 163-170.

17. H. K. Webb, V. K. Truong, J. Hasan, R. J. Crawford and E. P. Ivanova, J. Microbiol. Methods, 2011, 86, 131-139.

18. S. S. Hassani, M. Daraee, Z. Sobat, J. Adhes. Sci. Technol., 2020, 35, 221-241; DOI:10.1080/ 01694243.2020 .1798647$.

19. I. Sava, N. Hurduc, L. Sacarescu, I. Apostol and V. Damian, High Perform. Polym., 2013, 25, 13-24.

20. E. Sava, B. Simionescu, N. Hurduc and I. Sava, Opt. Mater., 2016, 53, 174-180.

21. I. Stoica, L. Epure, I. Sava, V. Damian and N. Hurduc, Microsc. Res. Tech., 2013, 76, 914-923.

22. I. Sava and T. Köpnick, Rev. Roum. Chim., 2014, 59, 583-590.

23. I. Sava, G. Lisa, E. Sava and N. Hurduc, Rev. Roum. Chim., 2016, 61, 419-426. 